Horizons philosophiques

\title{
Le corps et ses langages : quelques perspectives de travail historique
}

\section{Jean-Jacques Courtine}

Volume 1, numéro 2, printemps 1991

Sémiotiques 2 : théories et champs d'application

URI : https://id.erudit.org/iderudit/800869ar

DOI : https://doi.org/10.7202/800869ar

Aller au sommaire du numéro

Éditeur(s)

Collège Édouard-Montpetit

ISSN

1181-9227 (imprimé)

1920-2954 (numérique)

Découvrir la revue

Citer cet article

Courtine, J.-J. (1991). Le corps et ses langages : quelques perspectives de travail historique. Horizons philosophiques, 1(2), 1-11. https://doi.org/10.7202/800869ar d'utilisation que vous pouvez consulter en ligne.

https://apropos.erudit.org/fr/usagers/politique-dutilisation/ 


\section{Le corps et ses langages : quelques perspectives de travail historique}

Qu'on me permette pour commencer de dire en quoi la question du corps dans sa réalité historique s'est posée au cours d'une recherche qui avait été pour l'essentiel consacrée aux discursivités politiques. Cette brève relation personnelle d'un itinéraire n'a d'autre intérêt ici que d'introduire à des enjeux théoriques plus généraux : ceux d'un travail sur le rapport entre corps et discours dans la perspective d'une sémiologie historique d'une part, d'une histoire des pratiques langagières d'autre part. Le projet d'une analyse linguistique des discours politiques, tel qu'il s'était développé au sein de la linguistique en France à partir de la fin des années 60 , me semble avoir conduit pour une part à une impasse. Je ne peux détailler ici les raisons de cet échec ${ }^{1}$. Une trop grande importance accordée au texte au détriment des pratiques, aux systématicités linguistiques à l'encontre des événements historiques, à une logique stratégique de production des discours plutôt qu'aux effets dispersés de leur réception n'ont pas véritablement permis

1. Voir : J.J. Courtine, Corps et discours : éléments d'histoire des pratiques langagières et expressives, thèse d'État de linguistique, volume de présentation, Université de Paris- $X$, Nanterre, avril 1989, à paraître. 
de comprendre les mutations profondes qui ont affecté pendant la même période les discours politiques dans les sociétés occidentales ${ }^{2}$. Ces difficultés m'ont paru réclamer un "aggiornamento" théorique, en particulier une réorientation des perspectives historiques dans la saisie du discours; mais elles entraînent également tout un ensemble de conséquences empiriques : on ne peut plus, dorénavant, faire l'économie de l'analyse de représentations faites de discours, d'images et de pratiques. Qu'on songe simplement à la transmission de l'information politique par les médias, forme désormais dominante de communication politique : elle se donne comme un phénomène total de communication, représentation extrêmement complexe où les discours sont imbriqués dans des pratiques non verbales, où le verbe ne saurait être dissocié du corps et du geste, où l'expression par le langage se conjugue à celle du visage, où le texte est indéchiffrable en dehors de son contexte, où l'on ne peut plus séparer parole et image.

C'était ainsi, afin de comprendre les mutations récentes des discurvisités politiques, que l'idée s'est peu à peu imposée d'examiner le rapport entre corps et discours dans les formes de parole publique. Ce projet, d'abord circonscrit à l'époque contemporaine et à la sphère politique, s'est transformé, par la logique et l'historicité propres aux objets que l'on y rencontrait, en une enquête sur les pratiques et les représentations du visage et de l'expression depuis le XVIe siècle. Long détour, digression à l'origine imprévue, "accident» heureux de la recherche, qui a donné lieu à un ensemble de travaux d'anthropologie et

2. Ibid., chapitre III, p. 60-82; également : "Une politique du Çà, une politique du Moi», à paraitre dans M. Maillard, éd., L'impersonnel, Presses de l'Université de Grenoble III; “Les glissements du spectacle politique», Esprit, n० 164, sept. 1990 , p. 152-165. 
de sémiologie historiques du corps expressif, à une histoire du visage ${ }^{3}$.

II s'agissait à l'origine, à l'encontre de tant de commentaires sans mémoire voyant dans le calcul des apparences qui accompagne la parole publique contemporaine un mode de communication politique radicalement nouveau, de rappeler une longue tradition d'expression par le corps en même temps que de contrôle de celui-ci. "ll y a dans le visage une sorte d'éloquence silencieuse, qui, sans même agir, agit néanmoins", affirme ainsi le Père de Cressolles dans un traité de rhétorique jésuite composé au début du XVIIe siècle ${ }^{4}$. "La nature n'a pas seulement donné à l'homme la voix et la langue, pour être l'interprète de ses pensées (...). Elle a fait encore parler son front et ses yeux", ajoute Marin Cureau de la Chambre dans son Art de connaitre les hommes ${ }^{5}$. "ll est cependant à propos de composer son visage selon les circonstances où on se trouve et les personnes avec lesquelles on converse», recommande encore Jean Baptiste de la Salle dans son traité de civilité chrétienne ${ }^{6}$.

Tous ces textes le disent et le répètent : le visage parle. Par le corps, c'est l'individu qui s'exprime. Ou qui se tait.

3. Une dizaine d'articles et deux ouvrages, écrits en collaboration avec C. Haroche : une réédition de L'art de se taire (1771) de l'Abbé Dinouart, SainteAgnès, Éd. J. Millon, 1987; Histoire du visage (Exprimer et taire ses émotions $d u X V I^{e}$ au début du XIXe siècle), Paris, Rivages/Histoire, 1988.

4. Père de Cressolles, Vacationes Autumnales sive de perfecta oratoris actione et pronuntiatione, Paris, 1620; voir : M. Fumaroli, "Le corps éloquent...", XVII siècle, no 132, juillet/septembre 1981, p. 250.

5. M. Cureau de la Chambre, L'Art de connattre les hommes, Paris, 1659, p. 1.

6. J.B. de la Salle, Les règles de la bienséance et de la civilité chrétienne, Paris, 1703, p. 4. Les sources examinées incluent ainsi ouvrages de rhétorique et livres de civilité, mais aussi traités médicaux, manuels de déchiffrement du corps par la physionomie, arts de la conversation et du silence... 


\section{Les langages du visage :}

\section{une sémiologie historique de l'expression.}

Le projet a alors largement débordé le cadre initial de sa conception. Les perceptions du corps comme langage, les différentes sensibilités historiques aux expressions et aux silences du visage sont devenues le véritable objet de la recherche. Sans entrer dans le détail de ce travail, je voudrais simplement indiquer ici quelles perspectives il s'est efforcé d'ouvrir dans la voie d'une sémiologie historique du corps. Ce que fait apparaître en effet une histoire du visage, à travers le succès de la civilité, le renouveau de la physiognomonie et de l'actio rhetorica au cours du $X V^{\ominus}$ siècle, c'est tout d'abord ceci : avec la dissolution de la société médiévale, se dégage plus nettement un ensemble de savoirs et de pratiques - arts du langage, de la conversation, du corps, observations du visage et du geste, des contenances extérieures... - qui va vouloir substituer peu à peu aux forces obscures et aux marques gravées du destin, au silence de la présence divine, aux impulsions subites et inarticulées du corps, aux liens dictés par l'origine et aux échanges voulus par la tradition, la rationalité d'un paradigme de l'expression. Corps et langage y sont indissociables : autant que le verbe, le corps est expression subjective, lien social de communication, langage naturel de l'âme. Il est, comme le dit Cureau de la Chambre, "l'âme toute entière répandue au dehors". Le corps individuel est de plus en plus précisément perçu comme un discours, pénétré par une rationalité langagière qui donne sens à ses attitudes, ses gestes, ses postures. De ce langage du corps, l'Histoire du visage a tenté, en ce qui concerne l'expression faciale, de montrer les logiques et les transformations entre le $\left.X V\right|^{e}$ et le début du $X I X{ }^{e}$ siècle.

Ce processus de constitution du corps en discours à tenir, en texte à déchiffrer, a été en effet celui d'une progressive individualisation par l'expression. L'histoire de la 
physiognomonie révèle en particulier que les «lectures» du visage vont accorder une signification croissante aux signes mouvants et éphémères de l'expression quand elles ne se préoccupaient à l'origine que de traits stables et permanents de la figure : marques astrologiques, empreintes divines inscrites une fois pour toutes sur la physionomie. Graduellement, au cours des $X V l^{e}$ et $X V I I{ }^{e}$ siècles, tous les mouvements du corps individuel paraissent se charger de sens. Ils prennent valeur de signes à l'intérieur d'un code de communication tant verbal que corporel dont la nécessité partagée assure l'individualisation et la socialisation par l'expression des membres de la société civile. Ainsi, figures, postures et paroles sont-elles un lien social essentiel, mais aussi autant de traits individuels et singuliers. Au XVIIIe siècle, cette personnalisation du visage s'accroît encore : le discours du visage devient langage intérieur, fugace, sensible du sentiment. On y repère des indices de plus en plus subreptices, des mouvements de plus en plus ténus, d'éphémères frémissements. Le corps s'est lentement détaché des temps immobiles, des univers cosmologiques ou divins pour prendre sens dans une durée plus courte, dans un langage plus complexe et personnel. Un temps humain, un langage individuel : "Dans un individu, chaque instant a sa physionomie, son expression", dira alors Diderot.

Indépendamment de la discussion historique sur le bien-fondé de tels résultats, il est une perspective dont ce travail m'a paru indiquer tout à la fois l'importance et la nécessité : celle d'une sémiologie historique. Tout le monde s'accorde en effet sur l'idée que le corps "parle», que les gestes signifient, que le visage peut être un texte à interpréter. On a vu ainsi se multiplier ces dernières années des problématiques de la communication non verbale. II s'agit alors souvent de concevoir la dimension silencieuse des gestes, le discours muet des postures, le discret langage des figures et des mimiques à partir de présupposés 
éthologiques qui rapprochent l'observation des conduites humaines de celle du comportement animal, dans la voie jadis ouverte par Charles Darwin 7 . On recherche ainsi une "clé des gestes" qui conduit invariablement à l'idée d'une «nature» des comportements corporels, là où Marcel Mauss avait su déceler le caractère historique et social des constructions gestuelles ${ }^{8}$. Des perspectives anthropologiques en quête d'un codage universel de l'émotion dégénèrent alors en protocoles d'observation voulant traquer à fleur de peau des indices de vérité ou de mensonge 9 . On dispose encore de lourds codages para-linguistiques qui veulent épuiser la dimension énonciative de la "mimo-gestualité" 10; ou bien d'analyses micro-sociologiques qui assignent à la figuration du corps un sens dans le tissu serré des formes corporelles de la sociabilité, des ritualités gestuelles du quotidien ${ }^{11}$ : la précision du regard descriptif s'y paie par l'aplatissement de la profondeur historique. D'où viennent donc les gestes? Quelle est la genèse des expressions? Leur histoire est-elle vraiment sans importance lorsqu'on veut leur donner un sens?

Ces divers points de vue ont en commun, en dépit de leurs différences et de l'inégalité de l'intérêt qu'ils présentent, de vouloir élaborer des sémiologies du langage cor-

7. Ch. Darwin, The expression of emotions in man \& animals, London, 1872.

8. Par ex. D. Morris, La clé des gestes, Paris, Grasset, 1978, p. 8 : “Nous sommes des animaux. L'homo sapiens est une espèce de primate... La nature humaine n'est rien d'autre qu'une sorte particulière de nature animale".

9. Ainsi la dérive des travaux de P. Ekman, d'une discussion post-darwinienne sur l'universalité de l'expression des émotions à des manuels pratiques de "démasquage» destinés à dévoiler les mensonges; voir en particulier : Telling lies, clues to deceit in the marketplace, politics, and marriage, New York, Berkeley Books, 1985.

10. Voir par ex. J. Cosnier, "Communication et langage gestuels", in Les voies du langage, J. Cosnier \& al., Paris, Dunod, 1982.

11. E. Goffmann, Les rites d'interaction, Paris, Minuit, 1974. 
porel qui écartent la question de l'historicité de l'expression. Mais une sémiologie du corps comme système signifiant est-elle tout simplement possible en l'absence d'une sémiologie historique? Le déchiffrement du corps à partir de ses signes manifestes qu'effectue un devin ou un médecin antique, un physiognomoniste de l'âge classique, un naturaliste moderne, un psychanalyste, n'est en rien le même, bien qu'il puisse présenter des analogies très générales. Une histoire du visage le souligne : les marques gravées sur la physionomie que relève Jérôme Cardan vers le milieu du $X V I^{\ominus}$ siècle, les figures des passions que peint Charles Le Brun un peu plus d'un siècle plus tard, le vif mouvement des sentiments que veut saisir Lavater dans les années 1780 , le réflexe des émotions qu'observe Darwin vers la fin du XIXe siècle, et plus tard les symptômes d'un langage inconscient que Freud écoute sur le corps, ne sont pas des signes de même nature, ne permettent pas le même type d'interprétation. Ils ne supposent ni la même position pour qui les observe, ni la même identité de qui les produit.

De même, l'assertion vague et générale selon laquelle le visage "parlerait» prend sens dans le processus de longue durée où peu à peu le corps s'est constitué comme signe : livre tout d'abord où Dieu imprimait ses commandements, où les astres déposaient leur empreinte éternelle, où l'on pouvait discerner encore le reflet des ressemblances animales; puis rhétorique soumettant le corps à l'empire des figures et des postures; langage traduisant sur le visage la singularité, la sensibilité de l'individu; organisme s'exprimant dans la langue des réactions et des symptômes; discours enfin recherchant la problématique conjonction entre le sujet et son corps.

Le corps, le visage sont des objets historiques et culturels dont les perceptions sont liées, dans le registre de l'expression, aux représentations langagières, aux transformations de celles-ci. Et à suivre les mutations du régime 
du corps comme système signifiant se dégage, au point où le projet d'une histoire des mentalités croise celui d'une sémiologie générale, la perspective d'une sémiologie historique ${ }^{12}$. C'est là l'une des formes que peut revêtir la rencontre des préoccupations des historiens qui se sont donné le corps pour objet, et de ceux parmi les praticiens des sciences du langage qui ne considèrent pas le projet d'une sémiologie, inscrit dans la définition originelle de leur discipline, comme utopique, caduc, désormais étranger aux champs de leurs investigations; de ceux qui pensent encore que "le problème linguistique est avant tout sémiologique et (que) tous nos développements empruntent leur signification à ce fait important ${ }^{13}$ ".

\section{Conversation, silence :} une histoire des pratiques langagières.

L'expression par le corps et le visage est encore indissociable du langage dans ce qui va constituer le fondement de la société civile entre les $X V l^{\ominus}$ et $X V I I{ }^{\ominus}$ siècles : la pratique de la conversation.

Arts de la conversation, traités de cour, bienséances, manuels de civilité dessinent l'idéal et prescrivent les règles d'une société de langage dont jamais le corps ne s'absente. Société traversée d'injonctions paradoxales à bien des égards : la pratique de la conversation instaure peu à peu des réciprocités, l'exigence du dialogue s'y approfondit avec le sentiment des égards dus à autrui; l'homme qui converse se doit aux autres. Et cela ne va pas sans un

12. On en trouvera certains fondements théoriques dans le travail novateur de C. Ginsburg, "Signes, traces, pistes : les racines d'un paradigme de l'indice». Le Débat, no 6, nov. 1980. De nombreux travaux sur l'histoire du corps en développent depuis quelques années, implicitement ou explicitement, la perspective; on peut ici mentionner le récent ouvrage de J. C. Schmitt, La raison des gestes, Paris, Gallimard, 1990.

13. F. de Saussure, Cours de Linguistique Générale, Paris, Payot, 1971, p. 3435. 
contrôle renforcé, une vigilance accrue à l'égard des comportements et des contenances corporels. Mais en même temps l'individualité progressivement s'y affirme, les conduites se privatisent. L'homme qui converse se doit encore à lui-même. Ces exigences paradoxales s'inscrivent dans la matérialité des pratiques qui les réalisent, dans les paroles comme dans les gestes et les postures. Elles réclament de chacun une sorte de dédoublement psychologique qui tend à constituer les attitudes et les mouvements expressifs du corps en un discours continu, silencieux, parallèle à l'expression verbale, tout à la fois inséparable de cette dernière, mais pouvant s'y substituer dans ces pratiques de retrait en soi, présence minimale et muette de l'individu dans l'échange social par le langage.

"Je cognoy beaucoup de personnes qui ont une certaine vertu de savoir avec les yeux, la face, les gestes et autres signes extérieurs, à se montrer fort attentifs aux discours d'autruy et néanmoins ils pensent ailleurs de telle sorte qu'en un mesme point ils sont présents et absens et satisfont à un coup et aux autres et à eux-mesmes ${ }^{14}$,»

L'examen des pratiques de la conversation nous ramène ainsi à une histoire des techniques du corps expressif : il y a des manières de se taire qui sont des façons de parler ${ }^{15}$. Codifiés par l'actio rhetorica de l'éloquence antique qui renaît dans la rhétorique de la chair des années 1570 à 1625, postures, gestes et figures muettes du corps de l'orateur religieux vont progressivement se séculariser et inspirer les rituels profanes de la civilité. Ils expriment une exigence essentielle à la société civile : il faut savoir se contenir, se posséder, se taire, mais toujours porter sur le visage un air expressif. Bouhours a su condenser ce paradoxe dans le raccourci d'une formule : en société, «il

14. S. Guazzo, La civile conversation, Paris, 1592 (1574), p. 379.

15. Voir L'Art de se taire, op. cit. et Histoire du visage, op. cit., chapitre V. 
faut avoir la bouche fermée et le visage ouvert». En définitive, qu'ils proviennent de la tradition de civilité ou bien de celle des traités de cour, les préceptes de civilité interdisent de concevoir séparément les deux registres du corps et du langage, comme ceux du silence et de la parole. Le corps est discours; le silence est encore parole. C'est en cela que les "arts de se taire" demeurent des "arts de parler", qui, comme toute rhétorique, possèdent leurs principes et leurs règles.

Arts de la conversation, techniques du silence : deux objets d'une histoire des pratiques langagières, où à nouveau les pas des historiens et des linguistes peuvent se croiser. II ne s'agirait pas là d'histoire de la langue, bien que certains des faits que collecte cette dernière puissent y être précieux. II ne s'agirait pas non plus d'une histoire des théories linguistiques, orientation que suit pour l'essentiel et avec profit l'histoire de la linguistique. II s'agirait plutôt de penser, au sein de cette dernière, l'historicité des pratiques langagières où la linguistique rencontre certains de ces objets : le sujet de l'énonciation, la structure de l'interlocution, les actes de langage, la performativité, différentes formes de dialogisme, différentes situations, différents types de discours... Cette histoire pourrait être celle des manières de parler : histoire de l'énonciation et des positions qu'elle suppose, qui serait une histoire des conditions de possibilité des discours; histoire de la conversation et de ses dispositifs, qui rencontrerait celle de la constitution de la société civile, et qui contribuerait à la compréhension de la lente émergence de la société démocratique; histoire du corps expressif et de ses pratiques signifiantes, esquissée plus haut, qui serait l'un des éléments d'une archéologie des silences et des regards; histoire des institutions de langage, des techniques, des types de discours; histoire des sphères publique et privée de la parole, qui serait aussi celle du déplacement incessant de la frontière qui les départage... 
De cette histoire dont le domaine reste à inventorier et les limites à circonscrire, on peut trouver des éléments théoriques dans les travaux de N. Elias, M. Foucault, $J$. Habermas, ou bien dans ceux des historiens qui ont considérablement renouvelé l'histoire des mentalités, en explorant notamment les sensibilités du corps, les secrets de l'individu, les ritualités du quotidien, les formes de la vie privée... La participation à cet ensemble de travaux de ceux, parmi les linguistes, que l'histoire préoccupe, a été jusque là plutôt discrète. Elle est cependant nécessaire. L'heure des bilans me paraît en effet venue pour les sciences du langage : après les années de développement exceptionnel qu'elles ont connues durant le structuralisme, il est impossible de ne pas y déceler aujourd'hui, avec l'essoufflement des courants formalistes qui les dominent depuis lors, une perte de l'audience intellectuelle qui était la leur. Plus que jamais, une perspective qui donne au langage une inscription anthropologique et historique générale m'y semble nécessaire. C'est à cela que ces quelques pages ont voulu contribuer.

Jean-Jacques Courtine University of Southern California 